Goldschmidt 2021 Abstract

https://doi.org/10.7185/gold2021.4620

\section{Reactive Transport Modelling with a Coupled OpenFOAM®-PHREEQC platform}

SAIDEEP PAVULURI ${ }^{1}$, CYPRIEN SOULAINE ${ }^{2}$, FRANCIS CLARET $^{1}$ AND LAURENT ANDRE ${ }^{1,3}$

${ }^{1} \mathrm{BRGM}$

${ }^{2}$ Earth Sciences Institute of Orleans, Univ. Orleans, CNRS, BRGM

${ }^{3}$ ISTO

Presenting Author: saideep025@gmail.com

Fluid - mineral interactions are ubiquitous in a number of subsurface engineering applications, which include geothermal energy extraction, remediation of contaminants, evaluating the safety of sites that are used for the storage of nuclear wastes, carbon dioxide, and energy (like hydrogen gas). The ongoing chemical interaction between fluids and minerals results in the continuous evolution of porous media properties such as porosity, permeability, the reactive surface area that in turn influences the nature of fluid flows within the subsurface. To investigate such complicated hydro-geochemical systems, reactive transport modelling (RTM) has emerged as a key player to analyze the nature of fluid flows for various applications on spatio-temporal scales on par with subsurface applications.

We develop an open-source, generic, multi-scale package porousMedia4Foam [1] where we couple OpenFOAM ${ }^{\circledR}$ with PHREEQC to investigate hydro-geochemical interactions. The flow, transport of species, evolution of porous media properties, and temperature are handled by solving equations and various models implemented in OpenFOAM ${ }^{\circledR}$ whereas, the chemistry is exclusively handled by PHREEQC [2]. porousMedia4Foam has extensively been validated for single-phase flows at different scales i.e., at the pore-scale and continuum-scale for which reference solutions exist $[3,4]$. Additionally, we used porousMedia4Foam to investigate reactive transport mechanics in a hybrid setup comprising of a fracture sandwiched between reactive porous media (Figure 1).

Currently, the authors are extending the current capabilities of porousMedia4Foam by taking into account multiphase flow physics within a transport system with specific emphasis on analyzing the nature of fluid flow in geothermal production wells. This extension allows simulating phase changes of the hot geothermal fluids during their exploitation coupled with the chemical reactivity of the fluids (which may include $\mathrm{pH}$ variations, mineral precipitation in the well ...).

\section{Acknowledgments}

This study was done in the framework of the REFLECT Project, which has received funding from the European Union's Horizon 2020 research and innovation programme under grant agreement No 850626 .

\section{References}

[1] Soulaine et al. (2021). PorousMedia4Foam: Multi-scale open-source platform for hydro-geochemical simulations with OpenFOAM.
\& Appelo http://pubs.usgs.gov/tm/06/a43/

[3] Xie et al. (2015). Comput. Geosci. https://doi.org/10.1007/s10596-014-9458-3

[4] Poonoosamy et al. (2018). Comput. Geosci. https://doi.org/10.1007/s10596-018-9793-x

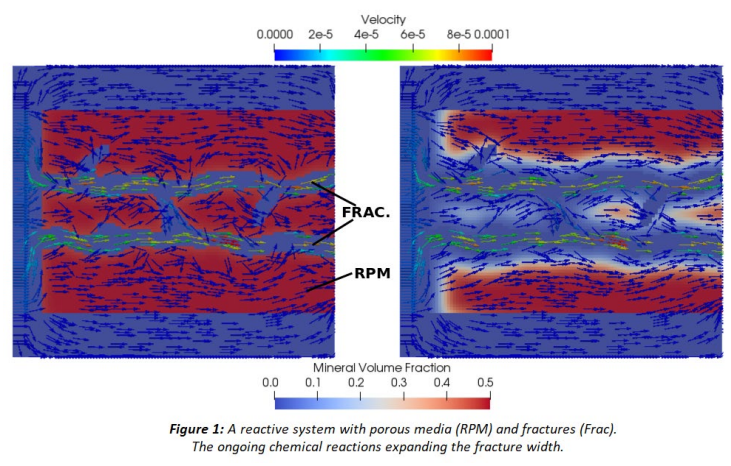

